Received: 17.10 .2020

Revised: 03.12.2020

Accepted: 18.12 .2020

DOI: $10.17804 / 2410-9908.2020 .6 .027-034$

\title{
A POSITRON ANNIHILATION STUDY OF DEFECT ACCUMULATION IN PHOSPHO- RUS- AND TITANIUM-ALLOYED AUSTENITIC STAINLESS STEELS UNDER ELEC- TRON IRRADIATION AT ROOM TEMPERATURE
}

\author{
D. A. Perminov \\ M.N. Miheev Institute of Metal Physics, UB RAS, \\ 18 S. Kovalevskoy St., Ekaterinburg, 620108, Russian Federation \\ iD https://orcid.org/0000-0003-1384-6446 ه d_perm@ramble.ru \\ Corresponding author. E-mail: d_perm@ramble.ru \\ Address for correspondence: ul. S. Kovalevskoy, 18, Ekaterinburg, 620108, Russian Federation \\ Tel.: (343) 37838 62; fax: (343) 3745244
}

The effect of phosphorus and titanium additions on the accumulation of vacancy defects in Cr16Ni15Mo3 austenitic stainless steels under electron irradiation at room temperature is studied by positron annihilation spectroscopy. It is shown that, at this temperature, phosphorus has no noticeable effect on the accumulation of vacancy defects. This is due to the low mobility of vacancies and the low concentration of impurities. Titanium, due to its high concentration, enhances the accumulation of vacancy defects during irradiation, but this effect is weak.

Keywords: austenitic stainless steels, phosphorus, titanium, electron irradiation, vacancy defects, positron annihilation.

\section{Acknowledgment}

The work was performed under the state assignment from the Ministry of Education and Science of Russi, theme Function No. AAAA-A19-119012990095-0, and partially supported by the RFBR, project No. 18-02-00270.

\section{Referencies}

1. Zinkle Steven J. and Busby Jeremy T. Structural materials for fission \& fusion energy. Materials Today, 2009, vol. 12, no. 11, pp. 12-19. DOI: 10.1016/S1369-7021(09)70294-9.

2. Zinkle S.J., Was G.S. Materials challenges in nuclear energy. Acta Materialia, 2013, vol. 61, pp. 735-758. DOI: 10.1016/j.actamat.2012.11.004.

3. Braislford A.D., Bullough R. Void growth and its relation to intrinsic point defect properties. Journal of Nuclear Materials, 1978, vol. 69-70, pp. 434-450. DOI: 10.1016/00223115(78)90259-3.

4. Watanabe H., Aoki A., Murakami H., Muroga T., Yoshida N. Effects of phosphorus on defect behavior, solute segregation and void swelling in electron irradiated Fe-Cr-Ni alloys. Journal of Nuclear Materials, 1988, vol. 155-157, pp. 815-822. DOI: 10.1016/0022-3115(88)90422-9.

5. Watanabe H., Muroga T., Yoshida N. The temperature dependent role of phosphorus and titanium in microstructural evolution of Fe-Cr-Ni alloys irradiated in FFTF. Journal of Nuclear Materials, 1996, vol. 228, pp. 261-274. DOI: 10.1016/0022-3115(96)80004-3.

6. Okita T., Wolfer W.G., Garner F.A., Sekimura N.Effects of titanium additions to austenitic ternary alloys on microstructural evolution and void swelling. Philosophical Magazine, 2005, vol. 85, no. 18, pp. 2033-2048. DOI: 10.1080/14786430412331331871. 
7. Odette G.R., Alinger M.J., Wirth B.D. Recent developments in irradiation resistant steels. Annual Review of Materials Research, 2008, vol. 38, pp. 471-503. DOI: 10.1146/ /annurev.matsci.38.060407.130315.

8. David C., Panigrahi B.K., Balaji S., Balamurugan A.K., Nair K.G.M., Amarendra G., Sundar C.S., Raj B. A study of the effect of titanium on the void swelling behavior of D9 steels by ion beam simulation. Journal of Nuclear Materials, 2008. vol. 383, pp. 132-136. DOI: 10.1016/j.jnucmat.2008.08.049.

9. $\quad$ Arunkumar J., Abhaya S., Rajaraman R., Amarendra G., Nair K.G.M., Sundar C.S., Raj B. Positron annihilation studies on D9 steel - Effect of Ti concentration. Physica Status Solidi (c), 2009, vol. 6, no. 11. pp. 2319-2321. DOI: 10.1002/pssc.200982097.

10. Perminov D.A., Druzhkov A.P., Pecherkina N.L., Arbuzov V.L. Accumulation of defects in austenitic stainless steels with phosphorus and titanium additions upon electron irradiation at $573 \mathrm{~K}$ investigated using positron annihilation spectroscopy. Physics of Metals and Metallography, 2019, vol. 120, no. 3, pp.284-289. DOI: 10.1134/S0031918X19030098.

11. Siegel R.W. Positron annihilation spectroscopy. Annual Review of Materials Science, 1980, vol. 10, pp. 393-425. DOI: 10.1146/annurev.ms.10.080180.002141.

12. Grafutin V.I., Prokop'ev E.P. Positron annihilation spectroscopy in materials structure studies. Physics-Uspekhi, 2002, vol. 45, no. 1, pp. 59-74. DOI: 10.3367/UFNr.0172.200201c.0067.

13. Morillo J., De Novion C.H., Dural J. Neutron and electron radiation defects in titanium and tantalum monocarbides: an electrical resistivity study. Radiation Effects and Defects in Solids, 1981, vol. 55, pp. 67-78. DOI: 10.1080/00337578108225467.

14. Druzhkov A.P., Perminov D.A., Pecherkina N.L. Positron annihilation spectroscopy characterization of effect of intermetallic nanoparticles on accumulation and annealing of vacancy defects in electron-irradiated Fe-Ni-Al alloy. Philosophical Magazine, 2008, vol. 88, pp. 959-976. DOI: $10.1080 / 14786430802014670$.

15. Arbuzov V.L., Druzhkov A.P., Danilov S.E. Effects of phosphorus on defects accumulation and annealing in electron-irradiated Fe-Ni austenitic alloys. Journal of Nuclear Materials, 2001, vol. 295, pp. 273-280. DOI: 10.1016/S0022-3115(01)00505-0.

16. Holzwarth U., Barbieri A., Hansen-Ilzhofer S., Shaaff P., Haaks M.Positron annihilation studies on the migration of deformation induced vacancies in stainless steel AISI 316L. Applied Physics A, 2001, vol. 73, pp. 467-475. DOI: 10.1007/s003390100781.

17. Druzhkov A.P., Arbuzov V.L., Perminov D.A. Positron annihilation study of effects of Ti and plastic deformation on defect accumulation and annealing in electron-irradiated austenitic steels and alloys. Journal of Nuclear Materials, 2005, vol. 341, pp. 153-163. DOI: 10.1016/j.jnucmat.2005.01.021. 
Подана в журнал: 17.10 .2020

УДК 539.12.043:620.192.63

DOI: $10.17804 / 2410-9908.2020 .6 .027-034$

\title{
ПОЗИТРОННЫЕ АННИГИЛЯЦИОННЫЕ ИССЛЕДОВАНИЯ НАКОПЛЕНИЯ ДЕФЕКТОВ В ЛЕГИРОВАННЫХ ФОСФОРОМ И ТИТАНОМ АУСТЕНИТНЫХ НЕРЖАВЕЮЩИХ СТАЛЯХ В ПРОЦЕССЕ ОБЛУЧЕНИЯ ЭЛЕКТРОНАМИ ПРИ КОМНАТНОЙ ТЕМПЕРАТУРЕ
}

\author{
Д. А. Перминов \\ ФГБУН Институт физики металлов им. М.Н. Михеева УрО РАН, \\ 18, ул. С. Ковалевской, Екатеринбург, Российская Федераџия \\ iD https://orcid.org/0000-0003-1384-6446 @ d_perm@ramble.ru \\ Ответственный автор. Электронная почта: d_perm@rambler.ru \\ Адрес для переписки: ул. С. Ковалевской, 18, 620108, Екатеринбург, Российская Федерация \\ Тел.: (343) 378-38-62; факс: (343) 374-52-44
}

\begin{abstract}
Работа посвящена исследованию влияние фосфора и титана на накопление вакансионных дефектов в аустенитных нержавеющих сталях X16Н15M3 при электронном облучении при комнатной температуре методом позитронной аннигиляционной спектроскопии. Показано, что при данной температуре фосфор не оказывает заметного влияния на накопление вакансионных дефектов. Это обусловлено малой подвижностью вакансий и низкой концентрацией примеси. Титан благодаря высокой его концентрации усиливает накопление вакансионных дефектов при облучении, однако этот эффект проявляется слабо.
\end{abstract}

Ключевые слова: аустенитные нержавеющие стали, фосфор, титан, электронное облучение, вакансионные дефекты, аннигиляция позитронов.

\section{1. Введение}

Конструкционные материалы, используемые в ядерных реакторах, работают в жестких условиях, сочетающих высокую температуру, коррозионное воздействие теплоносителя, механические напряжения, вибрацию и интенсивное облучение нейтронами. В этих условиях происходит интенсивная деградация материалов, что значительно ограничивает их использование. Поэтому существует острая необходимость в разработке материалов с улучшенными характеристиками, включая механическую прочность, достаточную пластичность, хорошую радиационную стойкость, а также устойчивость к коррозии $[1,2]$. Одним из материалов, наиболее полно удовлетворяющих указанным требованиям, являются аустенитные нержавеющие стали и сплавы. Эти сплавы проработаны с технологической точки зрения, обладают хорошими эксплуатационными характеристиками, сравнительно дешевы, их радиационная повреждаемость достаточно тщательно исследована. Однако аустенитные стали и сплавы подвержены радиационно-индуцированному распуханию (увеличению линейных размеров при облучении), что является главным фактором, ограничивающим их применение.

Одним из основных методов снижения вакансионного распухания сталей является легирование их малым количеством примесей. Взаимодействуя с точечными дефектами, атомы легирующих элементов образуют с ними комплексы примесь-вакансия и примесь-междоузельный атом и тем самым влияют на поведение точечных дефектов [3]. В качестве перспективных легирующих элементов, позволяющих снизить распухание сталей, рассмотрим титан и фосфор [4-6]. Так, например, титан, взаимодействуя с вакансиями, влияет на их подвижность и, соответственно, распухание сталей. Кроме того, в сталях, легированных титаном на уровне 1 ат. \%, в процессе облучения происходит распад твердого раствора и образование выделений вторых 
фаз (интерметаллидных выделений, карбидов титана), которые являются стоками или центрами усиленной рекомбинации для точечных дефектов [3, 6-9]. Добавка фосфора в аустенитную нержавеющую сталь также сдерживает вакансионное распухание под облучением [5-6]. Однако механизмы сдерживания развития пористости в стали при добавке фосфора до конца не изучены. При пониженных температурах фосфор, являясь подразмерной примесью, может взаимодействовать с межузельными атомами с образованием межузельных петель, которые являются стоками для мигрирующих вакансий [5]. Также в сталях возможно взаимодействие атомов фосфора с вакансиями [4]. Поскольку фосфор является быстродиффундирующей примесью, образование комплексов фосфор-вакансия приводит к увеличению эффективного коэффициента их диффузии и вероятности отжига вакансий на стоках. При повышенных температурах возможно образование фосфидов типа $\mathrm{Fe}_{2} \mathrm{P}$ и $\mathrm{Fe}_{3} \mathrm{P}$ игольчатой формы не когерентных матрице сталей [5]. Границы раздела «матрица-выделение» этих фосфидов содержат дислокации несоответствия, которые являются эффективными стоками точечных дефектов.

Ранее было исследовано влияние фосфора и титана на накопление вакансионных дефектов в аустенитных нержавеющих сталях на ранних стадиях облучения при температуре 573 К [10]. Цель работы - исследование этих эффектов при комнатной температуре облучения.

Для создания дефектов применялось облучение электронами высоких энергий. Электронное облучение, в отличие от нейтронного и ионного, генерирует свободно мигрирующие точечные дефекты (вакансии и межузельные атомы). Это позволяет изучать взаимодействие этих дефектов с примесями и несовершенствами кристаллической решетки стали.

Поведение дефектов при облучении исследовалось с помощью позитронной аннигиляционной спектроскопии (ПАС). Позитроны - хорошо известный зонд для дефектов вакансионного типа [11-12]. При этом ПАС обладает высокой чувствительностью к дефектам как по их размерам (от 0,1 до 3 нм), так и по концентрации $\left(10^{-3}-10^{-6}\right.$ на атом). Благодаря высокой чувствительности и избирательности к дефектам вакансионного типа ПАС позволяет исследовать поведение радиационных дефектов на начальной стадии радиационной повреждаемости (до $10^{-3}$ сна).

\section{2. Материалы и методика}

В работе исследовались аустенитные стали типа Х16Н15М3, легированные фосфором $(0,005$ мас. \% и 0,1 мас. \%) и титаном (1 мас. \%), выплавленные в вакуумной индукционной печи. Ленты из стали были прокатаны до толщины 150-200 мкм и нарезаны на пластины размером 10х10 мм². Полученные образцы после электролитической полировки отжигались при температуре $1323 \mathrm{~K}$ в атмосфере очищенного проточного гелия в течение 1 ч, а затем быстро закаливались в воду со скоростью $\approx 500 \mathrm{~K} / \mathrm{c}$.

Образцы сталей облучались на линейном ускорителе электронами с энергией 5 МэВ при температуре 293 К. Для обеспечения гомогенного облучения осуществлялось сканирование пучка электронов по поверхности образцов. Температура в процессе облучения поддерживалась с точностью $\approx 5 \mathrm{~K}$. Максимальный флюенс составлял $5 \cdot 10^{22}$ эл./ $\mathrm{m}^{2}$, что соответствует, согласно расчетам в рамках модифицированной модели Кинчина-Пиза [13], повреждающей дозе $\sim 5 \cdot 10^{-4}$ сна.

Образовавшиеся при облучении вакансионные дефекты диагностировались методом угловой корреляции аннигиляционного излучения (УКАИ), который является одной из методик ПАС [11-12]. УКАИ-метод был реализован на спектрометре с разрешением 1 мрад $\times 160$ мрад. В качестве источника позитронов использовался радионуклид ${ }^{68} \mathrm{Ge}$ активностью 400 МБк. В каждый спектр УКАИ набиралось около $5 \cdot 10^{5}$ отсчетов совпадений. Все измерения проводились при комнатной температуре. Спектры УКАИ представляют собой зависимость скорости счета совпадений от угла $\theta$ ( $\theta$ - отклонение угла разлета аннигиляционных $\gamma$-квантов от $\left.180^{\circ}\right)$. Угол $\theta=p_{z} / m_{0} c$, где $p_{z}$ есть поперечная компонента импульса электронно- 
позитронной пары; $m_{0}$ - масса покоя электрона; $c$ - скорость света в вакууме. Поскольку позитрон в образце термализован, то величина $\theta$ определяется импульсом аннигилирующего электрона. Таким образом, спектр УКАИ описывает распределение аннигилирующих электронов по импульсам. Подробно процедура обработки спектров УКАИ изложена в [14].

При захвате позитронов вакансионными дефектами или дислокациями происходят изменения в форме спектров УКАИ, поскольку электронная структура дефектов отличается от электронной структуры стали. Эти изменения характеризовались S-параметром, величина которого определяется как отношение площади под низкоимпульсной $\left(p_{z} \leq 3 \cdot 10^{-3} m_{0} c\right)$ частью спектра к полной площади под спектром. Изменения спектра УКАИ в этой области и, соответственно, S-параметра определяются концентрацией центров захвата позитронов следующим образом:

$$
S=\frac{\lambda_{f} S_{f}+\mu_{d} C_{0} S_{d}}{\lambda_{f}+\mu_{d} S_{d}}
$$

где $\lambda_{\mathrm{f}}-$ скорость аннигиляции позитронов в свободном (блоховском) состоянии; $\mu_{\mathrm{d}}-$ скорость захвата позитронов дефектами вакансионного типа; $C_{0}-$ концентрация вакансионных дефектов; $S_{\mathrm{f}}$ и $S_{\mathrm{d}}-$ значения $\mathrm{S}$-параметра, характеризующие аннигиляцию позитронов из свободного и захваченного дефектами состояния соответственно.

\section{3. Результаты и обсуждение}

На рис. 1 представлены зависимости S-параметра от флюенса электронов для стали $\mathrm{X} 16 \mathrm{H} 15 \mathrm{M} 3$ с различным содержанием фосфора, а также указана величина $S_{f}=0,526$, соответствующая аннигиляции позитронов из свободного (блоховского) состояния.

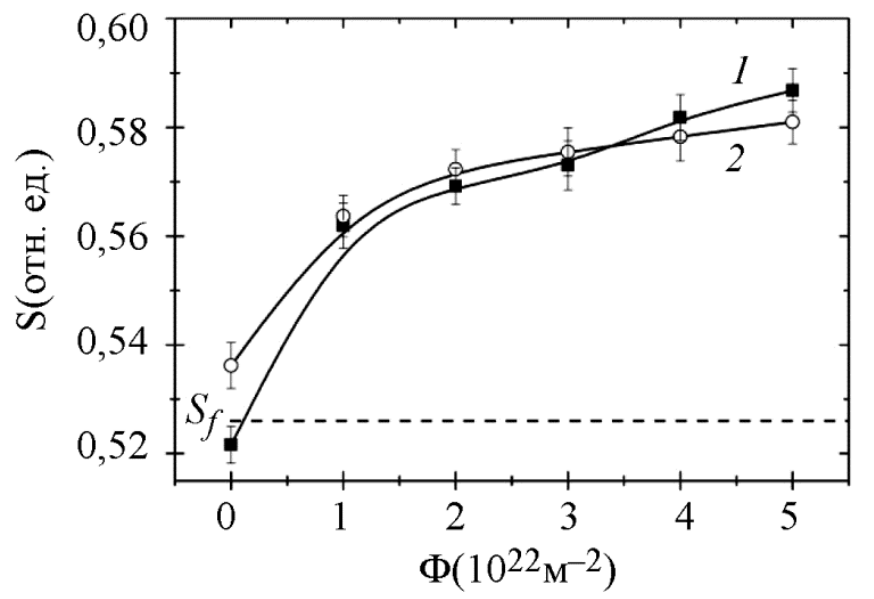

Рис. 1. Зависимость S-параметра от флюенса электронов для стали X16Н15M3 с различным содержанием фосфора: 1 - 0,005 мас. \% P; 2 - 0,1 мас. \% $\mathrm{P}$

Из рисунка следует, что исходное (до облучения) значение S-параметра для образца стали с содержанием фосфора 0,005 мас. \% совпадает с величиной $S_{f}$. Это указывает на то, что здесь отсутствуют какие-либо дефекты, способные захватывать позитроны (вакансии, вакансионные кластеры, дислокации и др.). Для стали, содержащей 0,1 мас. \% фосфора, исходное значение лежит несколько выше $S_{f}$, что свидетельствует о наличии в ней небольшого количества вакансий или дислокаций, образовавшихся при закалке. С ростом флюенса значение $\mathrm{S}$-параметра растет в обеих сталях, что обусловлено захватом позитронов дефектами вакансионного типа, образующимися при облучении. При этом кривые для обеих сталей близки. Из этого следует, что в сталях при облучении возникает одинаковое количество ва- 
кансий, т. е. фосфор при этой температуре облучения практически не влияет на накопление вакансионных дефектов.

На рис. 2 представлены зависимости S-параметра от флюенса электронов для стали $\mathrm{X} 16 \mathrm{H} 15 \mathrm{M} 3$, легированной титаном на уровне 1 мас. \%, с различным содержанием фосфора.

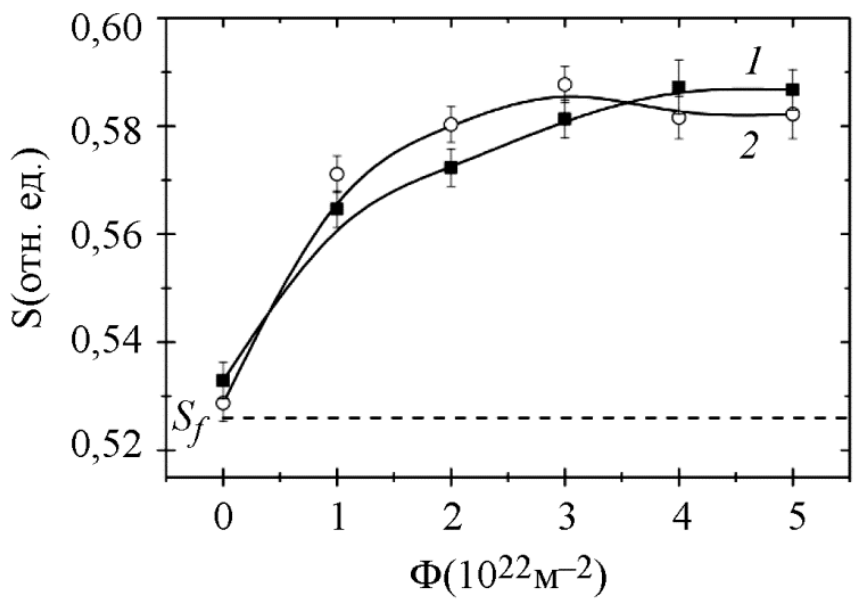

Рис. 2. Зависимость S-параметра от флюенса электронов для стали X16H15M3, легированной 1 мас. \% Тi, с различным содержанием фосфора: $1-0,005$ мас. \% P; 2 - 0,1 мас. \% P

Поведение S-параметра при облучении для этих сталей аналогично поведению $\mathrm{S}$-параметра для сталей, не содержащих титан. При этом существенных различий между кривыми для сталей с различным содержанием фосфора не наблюдается. Таким образом, в этих сталях при данной температуре облучения фосфор практически не влияет на накопление вакансионных дефектов. Следует отметить, что в сталях с титаном S-параметр при облучении выходит на тот же уровень, что и в сталях без титана $(0,586 \pm 0,002)$. В то же время, в сталях с титаном величина S-параметра достигает этого уровня при меньших значениях флюенса $\left(3-4 \times 10^{22} \mathrm{M}^{-2}\right)$, чем в сталях без титана. Таким образом, титан оказывает влияние на накопление вакансионных дефектов в процессе облучения при комнатной температуре.

Ранее исследовалось влияние фосфора и титана на накопление вакансионных дефектов в этих сталях при температуре облучения 573 К [10]. Было выявлено, что при данной температуре присутствие фосфора в сталях усиливает накопление вакансионных дефектов при облучении, что обусловлено взаимодействием атомов фосфора с вакансиями. Отсутствие эффекта фосфора при комнатной температуре обусловлено, по-видимому, низкой подвижностью вакансий при данной температуре. Энергия миграции вакансий в сталях составляет 0,9 эВ [15-16]. При комнатной температуре диффузионные длины для вакансий составляют единицы нм, поэтому лишь малая часть вакансий будет захвачена атомами фосфора. В силу этого эффект фосфора при данной температуре практически не заметен.

Титан, как и фосфор, также может взаимодействовать с вакансиями, усиливая их накопление при комнатной температуре [17]. При этом количество титана в стали на порядок превышает количество фосфора. Благодаря этому в сталях проявляется эффект титана на накопление вакансий при комнатной температуре, хотя он и выражен слабо.

\section{4. Заключение}

В работе методом позитронной аннигиляционной спектроскопии изучено влияние фосфора и титана на накопление вакансионных дефектов в аустенитных нержавеющих сталях на ранних стадиях облучения при комнатной температуре. Результаты работы показали, что при данной температуре фосфор не оказывает заметного влияния на накопление вакансионных дефектов. Это обусловлено малой подвижностью вакансий и низкой концентрацией 
примеси. Титан благодаря высокой его концентрации усиливает накопление вакансионных дефектов при облучении, однако этот эффект проявляется слабо.

\section{Благодарность}

Работа выполнена в рамках государственного задания МИНОБРНАУКИ России (тема «Функиия» № АAAA-A19-119012990095-0) при частичной поддержке РФФИ (проект № 18-02-00270).

\section{Литература}

1. Zinkle Steven J. and Busby Jeremy T. Structural materials for fission \& fusion energy // Materials Today. - 2009. - Vol. 12, iss. 11. - P. 12-19. - DOI: 10.1016/S1369-7021(09)70294-9.

2. Zinkle S. J., Was G. S. Materials challenges in nuclear energy // Acta Materialia. - 2013. Vol. 61. - P. 735-758. - DOI: 10.1016/j.actamat.2012.11.004.

3. Braislford A. D., Bullough R. Void growth and its relation to intrinsic point defect properties // Journal of Nuclear Materials. - 1978. - Vol. 69-70. - P. 434-450. - DOI: 10.1016/00223115(78)90259-3.

4. Effects of phosphorus on defect behavior, solute segregation and void swelling in electron irradiated Fe-Cr-Ni alloys / H. Watanabe, A. Aoki, H. Murakami, T. Muroga, N. Yoshida // Journal of Nuclear Materials. - 1988. - Vol. 155-157. - P. 815-822. - DOI: 10.1016/0022-3115(88)90422-9.

5. Watanabe H., Muroga T., Yoshida N. The temperature dependent role of phosphorus and titanium in microstructural evolution of Fe-Cr-Ni alloys irradiated in FFTF // Journal of Nuclear Materials. - 1996. Vol. 228. - P. 261-274. - DOI: 10.1016/0022-3115(96)80004-3.

6. Effects of titanium additions to austenitic ternary alloys on microstructural evolution and void swelling / T. Okita, W. G. Wolfer, F. A. Garner, N. Sekimura // Philosophical Magazine. 2005. - Vol. 85, no. 18. - P. 2033-2048. - DOI: 10.1080/14786430412331331871.

7. Odette G. R., Alinger M. J., Wirth B. D. Recent developments in irradiation resistant steels // Annual Review of Materials Research. - 2008. - Vol. 38. - P. 471-503. - DOI: 10.1146/ /annurev.matsci.38.060407.130315.

8. A study of the effect of titanium on the void swelling behavior of D9 steels by ion beam simulation / C. David, B. K. Panigrahi, S. Balaji, A. K. Balamurugan, K. G. M. Nair, G. Amarendra, C. S. Sundar, B. Raj // Journal of Nuclear Materials. - 2008. - Vol. 383. P. 132-136. - DOI: 10.1016/j.jnucmat.2008.08.049.

9. Positron annihilation studies on D9 steel - Effect of Ti concentration / J. Arunkumar, S. Abhaya, R. Rajaraman, G. Amarendra, K. G. M. Nair, C. S. Sundar, B. Raj // Physica Status Solidi (c). - 2009. - Vol. 6, no. 11. - P. 2319-2321. - DOI: 10.1002/pssc.200982097.

10. Accumulation of defects in austenitic stainless steels with phosphorus and titanium additions upon electron irradiation at $573 \mathrm{~K}$ investigated using positron annihilation spectroscopy / D. A. Perminov, A. P. Druzhkov, N. L. Pecherkina, V. L. Arbuzov // Physics of Metals and Metallography. - 2019. - Vol. 120, no. 3. - P. 284-289. - DOI: 10.1134/S0031918X19030098.

11. Siegel R. W. Positron annihilation spectroscopy // Annual Review of Materials Science. 1980. - Vol. 10. - P. 393-425. - DOI: 10.1146/annurev.ms.10.080180.002141.

12. Grafutin V. I., Prokop'ev E. P. Positron annihilation spectroscopy in materials structure studies // Physics-Uspekhi. - 2002. - Vol. 45, no. 1. - P. 59-74. - DOI: 10.3367/UFNr.0172.200201c.0067.

13. Morillo J., De Novion C. H., Dural J. Neutron and electron radiation defects in titanium and tantalum monocarbides: an electrical resistivity study // Radiation Effects and Defects in Solids. 1981. - Vol. 55. - P. 67-78. - DOI: 10.1080/00337578108225467.

14. Druzhkov A. P., Perminov D. A., Pecherkina N. L. Positron annihilation spectroscopy characterization of effect of intermetallic nanoparticles on accumulation and annealing of vacancy defects in electron-irradiated Fe-Ni-Al alloy // Philosophical Magazine. - 2008. Vol. 88. - P. 959-976. - DOI: 10.1080/14786430802014670. 
15. Arbuzov V. L., Druzhkov A. P., Danilov S. E. Effects of phosphorus on defects accumulation and annealing in electron-irradiated Fe-Ni austenitic alloys // Journal of Nuclear Materials. 2001. - Vol. 295. - P. 273-280. - DOI: 10.1016/S0022-3115(01)00505-0.

16. Positron annihilation studies on the migration of deformation induced vacancies in stainless steel AISI 316L / U. Holzwarth, A. Barbieri, S. Hansen-Ilzhofer, P. Shaaff, M. Haaks // Applied Physics A. - 2001. - Vol. 73. - P. 467-475. - DOI: 10.1007/s003390100781.

17. Druzhkov A. P., Arbuzov V. L., Perminov D. A. Positron annihilation study of effects of $\mathrm{Ti}$ and plastic deformation on defect accumulation and annealing in electron-irradiated austenitic steels and alloys // Journal of Nuclear Materials. - 2005. - Vol. 341. - P.153-163. DOI: 10.1016/j.jnucmat.2005.01.021. 